\title{
Review of: "Primary and Secondary Effects of Statin on Cerebro-Cardiovascular Disease Using the Nationwide Health Screening Data"
}

Jing $\mathrm{Wu}^{1}$

1 Tianjin University

Potential competing interests: The author(s) declared that no potential competing interests exist.

Summary $\square$ This study estimated the primary and secondary effects of statin on CVD among general population and the elderly subgroups by PSM. The results showed that statin had both primary and secondary prevention effects among general population, but there was only primary preventive effect on the elderly subgroups.

\section{Major Comments}

1. Introduction - Authors just explained why the study focused on the elderly population, but didn't clarify the reason why stratifying patients into primary and secondary prevention subgroups. Is there any evidence to support that these two subgroups were different?

2. Introduction - This section did not summarize the previous studies about the effects of statin on cardiovascular events were not summarized.

3. Materials and Methods - The information contained in the "National Health Insurance Service Health Screening Cohort Database" was not disclosed, e.g.: whether the database includes diagnostic information, prescription information and death information. And other data sources should be described if they were used.

4. Materials and Methods - The time design was unclear which made the study unreplicated. The definition of the index date of statin/non-statin users in primary and secondary prevention, and the length of baseline period should be disclosed. What's more, I suggest that the figure of study duration can be supplemented in the ESM. In addition, the select/monitoring period should be placed in "study population" section.

5. Materials and Methods - The definition of the MACE was not described. Besides, I want to know whether the cause of death can be identified in this database.

6. Materials and Methods - The analysis type (Intention-to-Treat, Per-Protocol or As Treated) is not mentioned, e.g.: how to deal with the patients who prescribed statin only once or twice in the follow-up period? If ITT analysis was adopted, the effects of statin could have been underestimated, which was a limitation that should be mentioned in the discussion section.

7. Materials and Methods - It seems that the variables in PSM were not well explained such as how these variables were chosen, and should the variables in the PSM be different between primary and secondary 
subgroups? What's more, more details about PSM should be exposed, e.g.: 1:1 match, caliber value and standardized mean difference (SMD).

8. Materials and Methods -Regression was not mentioned in statistical analysis section, but the HR value was reported in the results. If regression model was used, it should be added to and well described in the "method" section.

9. Results - The results of incidence of CVD mortality, fatal or nonfatal myocardial infarction (MI), and stroke were not reported, but they were mentioned in the method section.

10. Discussion - The discussion was insufficient. Authors just listed the results of previous studies in the literature review, with little explanation of the similarities and differences between this study and others. 